\title{
PTEN hamartoma tumor syndromes
}

The PTEN hamartoma tumor syndromes (PHTS) are a collection of rare clinical syndromes characterized by germline mutations of the tumor suppressor PTEN. These syndromes are driven by cellular overgrowth, leading to benign hamartomas in virtually any organ. Cowden syndrome (CS), the prototypic PHTS syndrome, is associated with increased susceptibility to breast, thyroid, and endometrial cancer. PTEN is located on chromosome 10q22-23 and negatively regulates the prosurvival PI3K/Akt/mTOR pathway through its lipid phosphatase activity. Loss of PTEN activates this pathway and leads to increased cellular growth, migration, proliferation, and survival. Clinical management of patients with PHTS, particularly those with CS, should include early and frequent screening, surveillance, and preventive care for associated malignancies. Concomitant with improved understanding of the biology of PTEN and the PI3K/Akt/mTOR pathway, inhibitors of this pathway are being developed as anticancer agents. These medications could have applications for patients with PHTS, for whom no medical options currently exist.

\section{In brief}

- PHTS is an autosomal dominant spectrum of hamartomatous overgrowth disorders with variable phenotypic manifestations characterized by germline mutations of the tumor suppressor gene PTEN located at 10q22-23.

- These syndromes include Cowden syndrome (CS), Lhermitte-Duclos disease (LD), Bannayan-RileyRuvalcaba syndrome (BRRS), and possibly Proteus syndrome (PS).

- The prevalence of identified germline PTEN mutations in these syndromes varies widely, with CS having 80\% prevalence of identified intragenic PTEN mutations, BRRS $65 \%$ prevalence, and PS less than 20\% prevalence.

- CS is a hamartomatous disorder characterized by macrocephaly, facial trichilemmomas, acral keratoses, papillomatous papules, and an increased risk for the development of breast, thyroid, and endometrial carcinoma.
- Adult onset LD is considered a variant of CS characterized by dysplastic gangliocytoma of the cerebellum often leading to increased intracranial pressure, ataxia, and seizures.

- BRRS is characterized by the developmental delay, macrocephaly, lipomas, hemangiomas, and pigmented speckled macules of the glans penis in males.

- PS is a complex, rapidly progressive disorder characterized by mosaicism, hemihypertrophy, subcutaneous tumors, and various bone, cutaneous and vascular anomalies. The association between PS and PTEN mutations remains controversial.

- Screening, surveillance, and preventive care for susceptible malignancies are the mainstays of clinical management for patients with PHTS.

- Because loss of PTEN increases activation of the PI3K/ Akt/mTOR pathway, drugs that target this pathway may have utility for treatment and/or prevention of tumors associated with PHTS.
Gideon M Blumenthal ${ }^{1}$ and Phillip A Dennis*,1

${ }^{1}$ Medical Oncology Branch, Center for Cancer Research, National Cancer Institute, Bethesda, MD, USA

European Journal of Human Genetics (2008) 16, 1289-1300; doi:10.1038/ejhg.2008.162; published online 10 September 2008

*Correspondence: Dr PA Dennis, Head, Signal Transduction Section, $\mathrm{NCl}$ / Navy Medical Oncology, Building 8, Room 5101, Medical Oncology Branch, Building 8, Room 5101, 8901 Wisconsin Avenue, Bethesda, MD 20889, USA. Tel: + 1301496 0929; Fax: + 1301496 0047;

E-mail: pdennis@nih.gov

Received 3 April 2008; revised 9 July 2008; accepted 6 August 2008; published online 10 September 2008

\section{Introduction}

The classification of the PTEN hamartoma tumor syndromes (PHTS) was developed as a means of unifying the disparate disorders associated with germline mutations in the tumor suppressor gene PTEN (phosphatase and tensin homolog, deleted on chromosome 10). ${ }^{1,2}$ PTEN encodes a dual phosphatase protein that negatively regulates the PI3K/Akt/mTOR pathway. Somatic loss of PTEN function through mutation, deletion, or methylation has been described in various sporadic human cancers, including those of the brain, bladder, breast, prostate, colon, lung, and endometrium, ${ }^{3}$ and is thus under intense investigation by cancer researchers. 
PTEN hamartoma tumor syndromes GM Blumenthal and PA Dennis

1290

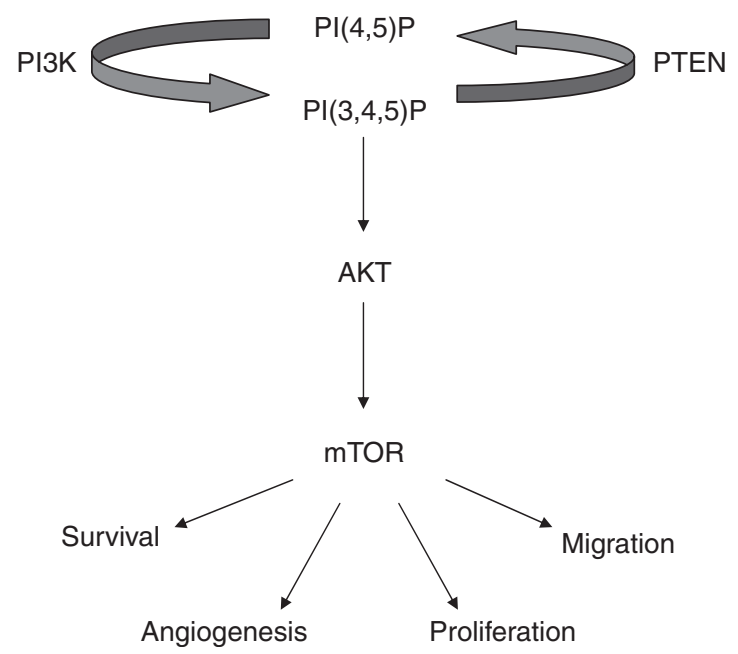

Figure 1 Schematic representation of the PI3K/Akt/mTOR pathway.

The PHTS are a spectrum of syndromes with variable clinical manifestations characterized by aberrant growth and associated with germline PTEN mutations. Hamartomas are a histologically distinct subtype of benign tumors in which cells maintain normal differentiation but are disorganized with respect to architecture. Cowden syndrome (CS) is the prototypic syndrome, characterized by mucocutaneous lesions, benign hamartomas, macrocephaly, and increased predisposition to breast, thyroid, and endometrial carcinoma. Lhermitte-Duclos (LD), a variant of CS, is characterized by dysplastic gangliocytomas of the cerebellum, which can lead to hydrocephalus, ataxia, and seizures.

After the discovery of the PTEN gene and the fact that CS is caused by germline mutations of PTEN, it became apparent that CS is allelic to other seemingly unrelated clinical syndromes. Bannayan-Riley-Ruvalcaba syndrome (BRRS), characterized by the developmental delay, macrocephaly, lipomas, hemangiomas, and speckled penis in males, is associated with PTEN mutations in approximately $60 \%$ of cases. Proteus syndrome has also been associated with germline PTEN mutations, although this is controversial. The clinical management of PHTS patients has historically focused on genetic counseling and screening. Indeed, patients with PHTS, particularly those with CS, should undergo early and frequent surveillance for susceptible malignancies. No medical therapies currently exist for PHTS patients. Because loss of PTEN increases activation of the PI3K/Akt/mTOR pathway, and inhibitors of this pathway are being developed as anticancer agents, these drugs might have therapeutic efficacy in patients with PHTS, which will be discussed further in this review.

\section{PTEN biology}

The PTEN gene (also known as MMAC1 for mutated in multiple advanced cancers, or TEP1 for TGF- $\beta$ regulated and epithelial cell-enriched phosphatase) spans nine exons and is located on chromosome 10q22-23. The gene encodes a 403 amino-acid protein, which acts as a dualspecificity phosphatase that dephosporylates lipids and proteins. PTEN exerts its lipid phosphatase activity by dephosphorylating the 3 '-phosphoinositide products of PI3K, causing conversion of phosphatidylinositol $(3,4,5)$ trisphosphate to phosphatidylinositol $(4,5)$ bisphosphate and conversion of phosphatidylinositol $(3,4)$ bisphosphate to phosphatidylinositol (4) phosphate. Reduction of 3 '-phosphoinositides decreases activity of kinases downstream of PI3K such as phosphoinositide-dependent kinase 1, Akt, and mTOR, and is responsible for its tumor suppressor activity. Because of negative regulation of the Akt pathway, PTEN indirectly decreases phosphorylation of other substrates downstream of Akt such as p27, p21, GSK-3, Bad, ASK-1, as well as members of the forkhead transcription factor family (eg, AFX, FKHR, FKHRL1). Thus, a loss or reduction in PTEN activity leads to increased phosphorylation of many key cellular proteins, which in turn can affect processes such as cell cycle progression, metabolism, migration, apoptosis, transcription, and translation (Figure 1).

PTEN also has activities independent of its lipid phosphatase activity. As a protein phosphatase, PTEN dephosphorylates focal adhesion kinase, which can inhibit cell migration and cell spreading. ${ }^{4}$ The protein phosphatase activity of PTEN can also regulate the mitogen-activated protein kinase pathway, thus regulating cell survival. ${ }^{5,6}$ Shen et $\mathrm{al}^{7}$ demonstrated that PTEN can promote chromosomal stability independent of its lipid phosphatase activity. ${ }^{7}$ Targeted mutations of PTEN in its carboxyterminal domain, but not its phosphatase domain, caused centromere breakage and chromosomal translocations. Because mutations can occur throughout the PTEN gene, it is possible that chromosomal instability is characteristic of tumors derived from PHTS patients with mutations encoding the carboxy terminus of the protein.

In mouse models, complete inactivation of both copies of the PTEN gene before embryonic day 13.5 results in embryonic death because of aberrant differentiation and patterning of the germ layers. PTEN-null embryos exhibit poorly organized ectodermal and mesodermal layers, and overgrowth of the cephalic and caudal regions. ${ }^{8,9}$ Monoallelic inactivation, however, results in PTEN heterozygous knockout mice that are able to complete embryogenesis. Several features observed in PTEN heterozygous knockout mice are similar to those seen in PHTS syndromes. PTEN heterozygote mice develop neoplasms in many organs, including the liver, prostate, endometrium, GI tract, thymus, and thyroid. ${ }^{10}$ Conversely, several groups have demonstrated that reconstitution of wild-type PTEN into a mutant PTEN background decreases activation of the PI3K/ Akt/mTOR pathway, alters cell cycle distribution, and/or induces apoptosis. ${ }^{11-13}$ These heterozygote knockout mice 
serve as potential animal models for PHTS, which will facilitate the development of novel therapies for patients with PHTS.

\section{Overview of syndromes \\ Cowden syndrome}

Cowden syndrome was first described by Lloyd and Dennis ${ }^{14}$ in 1963 in a case report of a patient with multiple hamartomas, unique mucocutaneous findings, fibrocystic breast disease, and abnormal neurologic findings. CS is an autosomal dominant disorder with age-related penetrance characterized by malignant and benign hamartomatous lesions affecting derivatives of all three germ cell layers. Since the identification of the susceptibility gene PTEN, the estimated incidence of CS increased from 1 in 1000000 to 1 in 200000 individuals. This most likely remains an underestimation, as CS is associated with a high degree of phenotypic variability and its hallmark features are under-recognized within the medical community. ${ }^{15}$

In patients with CS, hamartomas can be found in virtually every organ, but most commonly in the skin and gastrointestinal (GI) tract. Mucocutaneous features, including trichilemmomas and papillomatous papules, are estimated to have a $99 \%$ penetrance in CS by the third decade of life. ${ }^{1}$ Trichilemmomas are flesh-colored hamartomas of the outer sheath of the follicle that are typically near the hairline (Figure 2a). Papillomatous papules are mucocutaneous lesions found on the face (Figure 2c), oral mucosa, and acral surfaces (Figure 2b).

Gastrointestinal polyps in CS are typically asymptomatic, and can occur anywhere in the GI tract, with colonic polyps present in $60-90 \%$ of patients. ${ }^{16}$ The upper GI lesions are also relatively common (Figure 2d), including glycogenic acanthosis, characterized by raised greywhite plaques in the distal esophagus, comprised of glycogen-filled squamous cells. ${ }^{17}$ Despite the fact that the two most common sites for hamartomas in CS are the skin and GI tract, there is no definitive evidence that patients with CS are at increased risk to develop dermatologic or GI malignancies.

Benign thyroid lesions occur in up to $75 \%$ of patients with CS, including adenomas, hamartomas, multinodular goiter, and Hashimoto's thyroiditis. ${ }^{16,18}$ Up to half of women with CS are afflicted with benign breast disease, which can be extensive and bilateral. ${ }^{19}$ In addition,
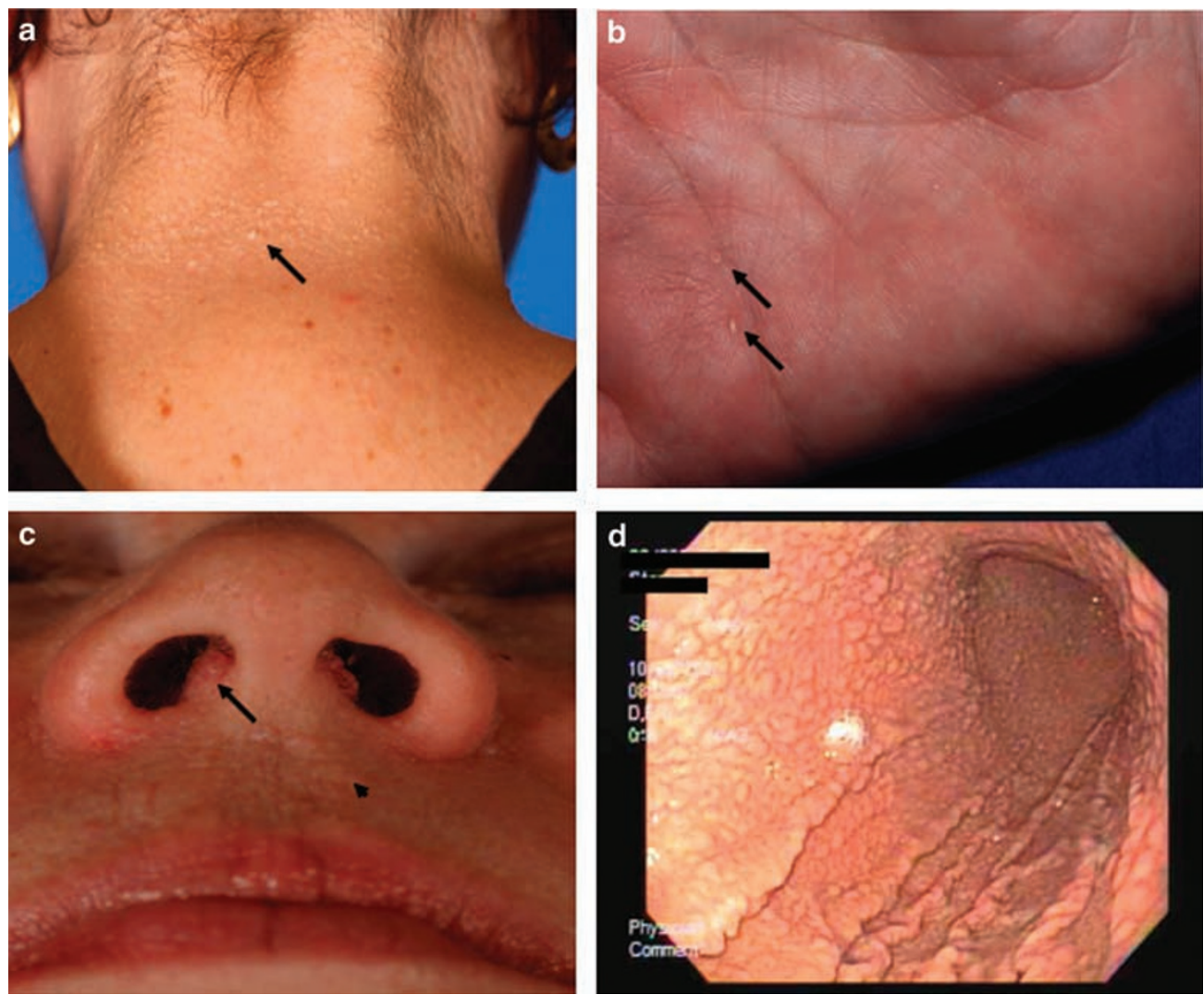

Figure 2 Pathognomonic mucocutaneous features of Cowden syndrome (CS). (a) Trichilemmomas on the nape of the neck of a subject with CS. (b) Palmar keratoses in a subject with CS. (c) Perioral papillomatous papules (arrow head) and nasal polyposis. (d) Gastric hamartomas as seen by endoscopy in a subject with CS. 
Table 1 PTEN hamartoma tumor syndromes (PHTS)

\begin{tabular}{|c|c|c|c|}
\hline Syndrome & $\begin{array}{l}\text { Prevalence of } \\
\text { PTEN mutation }\end{array}$ & Clinical manifestation & Associated malignancies \\
\hline Cowden syndrome (CS) & $80 \%$ & $\begin{array}{l}\text { Trichilemmomas, mucocutaneous papillomatous } \\
\text { papules, macrocephaly }\end{array}$ & $\begin{array}{l}\text { Breast, thyroid, endometrial } \\
\text { carcinoma }\end{array}$ \\
\hline Lhermitte-Duclos disease (LD) & $83 \%$ & Ataxia, increased intracranial pressure, seizures & None demonstrated \\
\hline $\begin{array}{l}\text { Bannayan-Riley-Ruvalcaba } \\
\text { syndrome (BRRS) }\end{array}$ & $60 \%$ & $\begin{array}{l}\text { Macrocephaly, developmental delay, intestinal } \\
\text { hamartomas, lipomatosis, hemangiomatosis, } \\
\text { speckled penis in males }\end{array}$ & None demonstrated \\
\hline Proteus syndrome (PS) & $? ?$ & $\begin{array}{l}\text { Congenital malformations, tissue overgrowth, } \\
\text { epidermal nevi, hyperostosis }\end{array}$ & None demonstrated \\
\hline
\end{tabular}

approximately half of women develop uterine leiomyomas or fibroids, typically by the third decade of life. ${ }^{20}$ Other genitourinary malformations described in CS patients include horseshoe kidney and bicornate uterus.

Lipomas, fibromas, and vascular abnormalities are also characteristic of CS. Tan et $a,^{21}$ through analysis of angiographic imaging studies, recently characterized the vascular anomalies in patients with PHTS (CS and BRRS) and found such abnormalities in 54\% of patients, mainly multifocal intramuscular combinations of fast-flow channels and ectopic fat, as well as intracranial developmental venous anomalies. Other central nervous system abnormalities include macrocephaly, present in up to $100 \%$ of CS patients, ${ }^{22}$ and dysmorphology of the head. The developmental delay has been described with CS, although not as commonly as with BRRS or PS.

Cowden syndrome patients are at increased risk to develop breast, thyroid, and endometrial cancer. The lifetime risk of breast cancer in women with CS is estimated to be as high as $50 \%,{ }^{16,19,23}$ as compared to $11 \%$ within the general population. As with other hereditary breast cancer syndromes, diagnosis of breast cancer occurs at a younger age compared to the general population, with the average age of breast cancer diagnosis 36-46 years. ${ }^{24}$ The predominant breast cancer histology is invasive ductal adenocarcinoma, although lobular and tubular carcinomas have also been observed. Schrager et $a l^{19}$ studied the histopathology of breast biopsies in 19 unrelated CS patients and found that in some cases malignant tumor was contained within densely fibrotic, hamartomatous regions within the breast, suggesting that these invasive carcinomas may arise within benign hamartomas. On the basis of case reports, males with CS also may be at increased risk of breast cancer, although risk assessment is difficult to quantify because of the rarity of male breast cancer. ${ }^{25}$

The lifetime risk of thyroid cancer in both genders is estimated to approach $10 \%$, as compared to less than $1 \%$ in the general population. The predominant thyroid cancer histology is follicular, although papillary thyroid cancers are observed as well. Endometrial cancer is more frequent in women with CS with an estimated risk of $5-10 \%$ as compared to $<2.5 \%$ in the general population, although this risk estimate in CS needs to be formally verified. ${ }^{26}$ In addition to breast, thyroid, and endometrial cancers, other malignancies have anecdotally been reported to be increased with $\mathrm{CS}$, including melanoma, renal cell carcinoma, and gliomas. ${ }^{1}$

Germline mutations resulting in loss of function of the PTEN gene are found in approximately $80 \%$ of patients with CS (Table 1), with approximately $40-60 \%$ of cases estimated to be familial. ${ }^{2,27}$ Approximately two-thirds of mutations in CS occur in exons 5, 7, and 8 of the PTEN gene. Exon 5, which encodes the PTEN phosphatase core motif and comprises merely $20 \%$ of the PTEN gene, is associated with approximately $40 \%$ of identified mutations in CS. $^{2}$ Approximately $20 \%$ of CS patients meet clinical diagnostic criteria but have no identified exonic or splice site mutation. Zhou et $a l^{28}$ demonstrated that approximately $10 \%$ of these patients have mutations in the promoter region of PTEN, resulting in decreased PTEN protein levels and increased expression of phosphorylated Akt. Nevertheless, approximately $18 \%$ of CS patients have no identified genetic explanation for their phenotypic features. Whether these patients' phenotypes can be explained by inactivation of PTEN through other mechanisms such as expression of specific microRNAs, ${ }^{29}$ overexpression of the NEDD4 E3-ubiquitin protein that leads to degradation of PTEN in the proteasome, ${ }^{30,31}$ PTEN promoter methylation, ${ }^{32}$ or splice site variability ${ }^{33,34}$ is unclear.

In 1995, the International Cowden Consortium established a set of strict operational diagnostic criteria for CS on the basis of published data and expert opinions, which facilitated the identification of the susceptibility gene on chromosome 10q22-23. The diagnostic criteria were revised in $2000^{1}$ and in 2004, and are now updated annually by the US National Comprehensive Cancer Network (Table 2). In any patient who meets the pathognomonic criteria for CS, referral to a genetic counselor should be pursued and sequencing of PTEN should be considered. For patients who meet clinical criteria for CS but in whom sequencing of the coding region does not reveal a mutation in PTEN, further analysis of PTEN (eg, sequencing of the promoter region, assessment of PTEN protein levels) could be considered, but these approaches 
Table 2 International Cowden Consortium Diagnostic Criteria

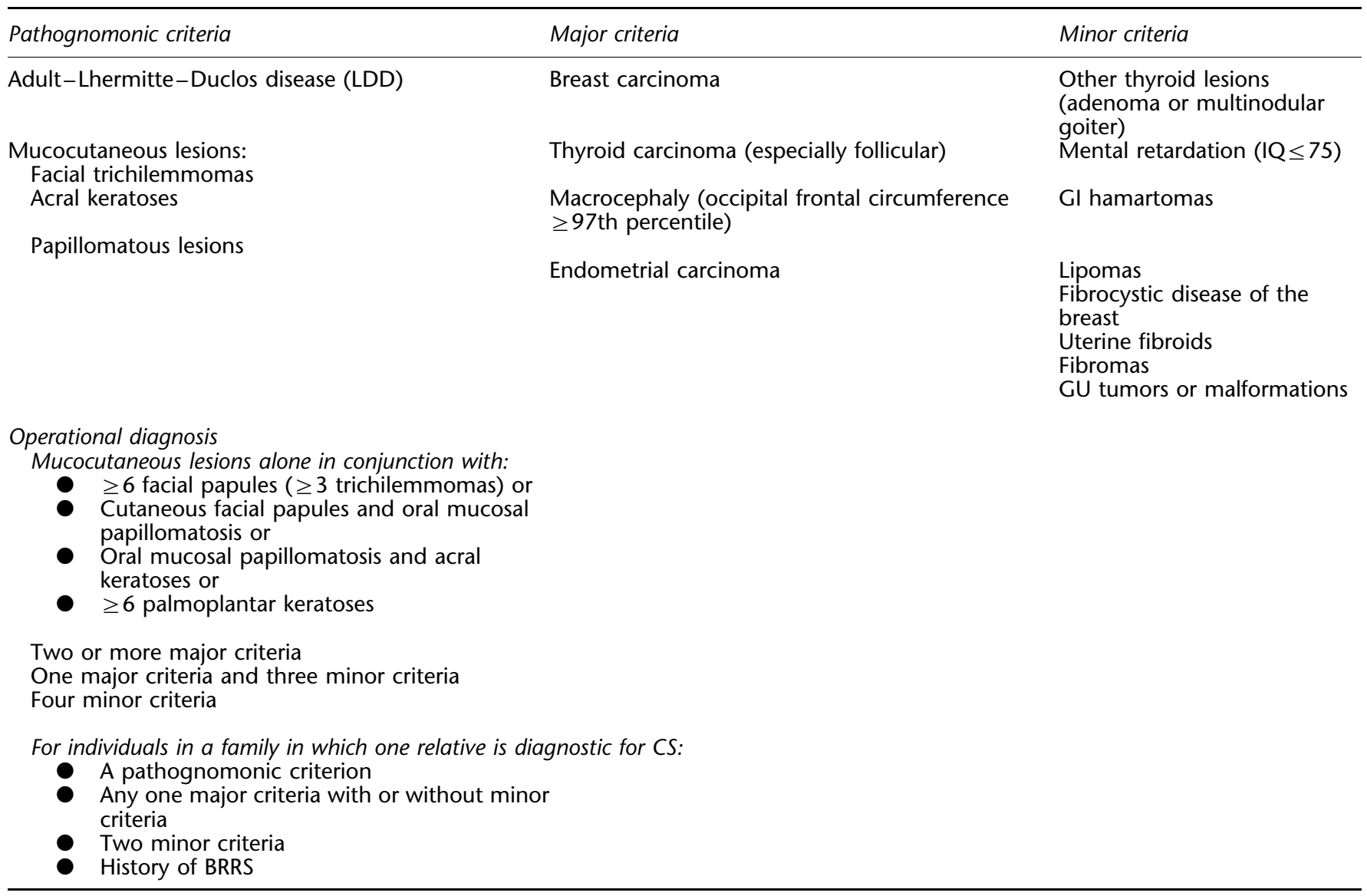

are currently experimental. A general diagnostic algorithm for CS is outlined in Figure 3. With the expanding knowledge of the phenotypic manifestations of germline PTEN mutations, the diagnostic criteria will ultimately be refined to enable simpler diagnosis, thus increasing the likelihood of capturing patients with CS and ensuring that appropriate management is instituted. ${ }^{35}$

\section{Lhermitte-Duclos disease}

Lhermitte-Duclos, or dysplastic gangliocytoma of the cerebellum, is considered a phenotypic variant of CS. In 2004, LD was recognized as a pathognomonic criterion for CS by International Cowden Consortium Criteria. Adult onset LD is characterized by dysplastic expansion of ganglion cells within the cerebellum, leading to replacement of the cerebellar internal granule cell layer. Zhou et $a l^{36}$ demonstrated that 15 out of 15 adult-onset LD samples had PTEN mutations, whereas no mutations were found in specimens from pediatric patients with LD. This suggests that pathogenesis of childhood variant LD may not be related to mutated PTEN. The presentation of LD is highly variable. The more severe form can be life- threatening with increased intracranial pressure, ataxia, and seizures. The treatment for symptomatic cerebellar gangliocytoma is surgical resection; however, regrowth of LD lesions following resection has been reported. ${ }^{37}$ Abel et $a l^{38}$ studied cerebellar lesions from 31 LD cases. The authors noted that in addition to hypertrophic ganglionic cells, histopathologic analysis revealed prominent vascular proliferation and white matter vacuolization. The investigators also found absent PTEN protein expression in $66 \%$ of samples analyzed, activated Akt in 90\% of samples analyzed, and increased phosphorylation of S6, a downstream substrate of mTOR, in all samples analyzed.

\section{Bannayan-Riley-Ruvalcaba syndrome}

Originally described in 1971, ${ }^{39}$ BRRS has also been known by other names, including Bannayan-Zonana syndrome (BZS), Ruvalcaba-Mhyre syndrome, and Riley-Smith syndrome. BRRS is characterized by macrocephaly, benign hamartomas, pigmented macules of the glans penis, lipomas, hemangiomas, and the developmental delay, or mental retardation. Other phenotypic features of BRRS disorder include thyroid abnormalities such as 

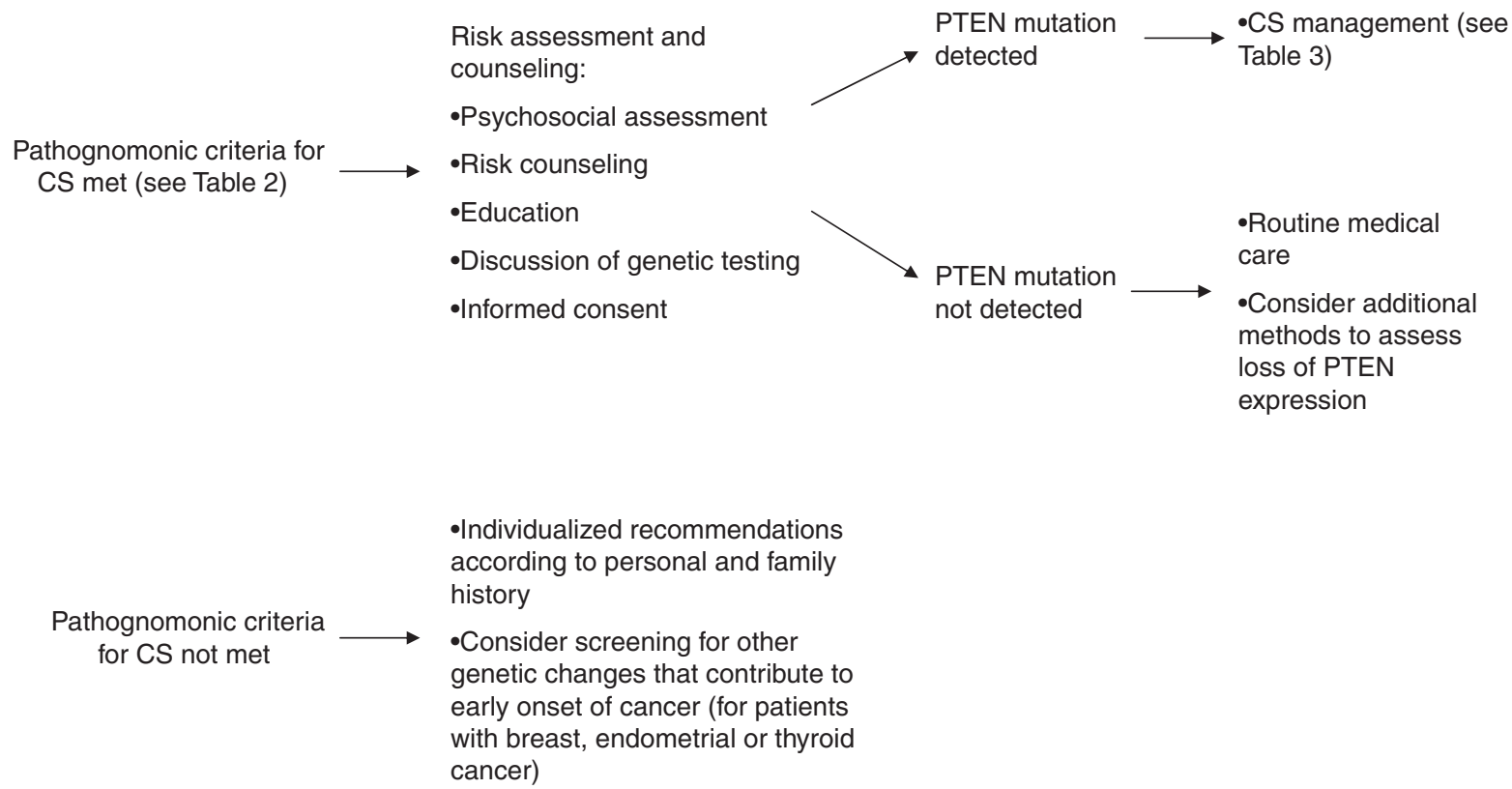

Figure 3 Diagnostic algorithm for Cowden syndrome.

Hashimoto's thyroiditis, high-arched palate ${ }^{40-43}$ overgrowth of prenatal or postnatal onset, macrosomia, hypotonia, joint hyperextensibility, downward slanting palpebral fissures, frontal bossing, hypoglycemia, seizures, and café au lait spots. These phenotypic features are highly variable, although they appear to cluster within a given family.

Although there are no international consensus criteria for the diagnosis of BRRS, several groups of investigators have proposed criteria to facilitate clinical diagnosis. Marsh et $a l^{2}$ defined a clinical diagnosis of BRRS as meeting three out of four features: macrocephaly, lipomatosis, hemangiomas, and speckled penis in males. Parisi et al ${ }^{43}$ used less stringent criteria, defining the syndrome as having two out of three of the following features: macrocephaly, hamartomas (including at least one lipoma, hemangioma, or intestinal polyp), and penile macules in males. BRRS appears to have a male predominance, although this may be confounded by a diagnostic bias in that a speckled penis is considered pathognomonic for the syndrome. In contrast, CS appears to have a bias toward increased diagnosis in women, given that breast and endometrial cancer comprise major diagnostic criteria. In addition, the BRRS phenotype appears to have an earlier age-related penetrance as compared to CS.

After the discovery of the PTEN gene on chromosome 10q22-23, BRRS was found to be allelic to CS, with approximately $60 \%$ of BRRS patients having identified

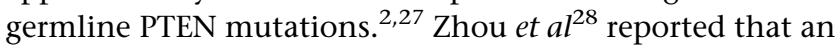
additional $11 \%$ of BRRS-related mutations include large deletions, often favoring the $5^{\prime}$ end of the gene containing exon 1 and the promoter region. There is significant phenotypic overlap between CS and BRRS with common features including hamartomas, macrocephaly, and thyroid abnormalities. Interestingly, identical PTEN mutations have been found in patients who present with phenotypic manifestations characteristic of either BRRS or CS. Even individuals within a single family that have the same germline PTEN mutation can have phenotypic features more consistent with either BRRS or CS. Recently, Lachlan et al $^{22}$ studied genotype-phenotype correlations in a cross-sectional study of 42 patients with known PTEN mutations from 26 different families. The earliest phenotypic features among affected individuals were macrocephaly and hamartomas, with mucocutaneous features and malignancies developing in some patients later in adulthood. These authors ${ }^{22}$ and others ${ }^{27}$ have suggested that BRRS and CS represent a single disorder with variable phenotypic expression and age-related penetrance, and have questioned whether the distinction between BRRS and CS is clinically relevant.

Before it was recognized as allelic to CS, BRRS was not considered to cause increased risk of malignancy. However, in a study of families with BRRS and CS overlap, a correlation between benign and malignant breast disease and germline PTEN mutation was observed, raising the possibility that BRRS is indeed associated with increased susceptibility to malignancy. ${ }^{27}$ Given the genotypic and phenotypic similarities between BRRS and CS, and the possibility that these syndromes may represent a single disorder, BRRS patients should be counseled on adhering to screening guidelines recommended to CS patients (Table 3). 
Table 3 Screening Guidelines Advocated by NCCN 2007

\begin{tabular}{|c|c|c|}
\hline Type of screening & When? & How often? \\
\hline \multicolumn{3}{|l|}{ Women } \\
\hline Breast self-exam & Age 18 years & Monthly \\
\hline Clinical breast exam & $\begin{array}{l}\text { Age } 25 \text { or } 5-10 \text { years before earliest known breast } \\
\text { cancer in the family. }\end{array}$ & Every 6 months \\
\hline Mammogram and breast MRI & $\begin{array}{l}\text { Age } 30-35 \text { or } 5-10 \text { years before earliest known } \\
\text { breast cancer in the family. }\end{array}$ & Every 12 months \\
\hline Blind endometrial biopsy & $\begin{array}{l}\text { Age } 30-35 \text { or } 5 \text { years before earliest diagnosis of } \\
\text { endometrial cancer in family until menopause }\end{array}$ & Every 12 months \\
\hline Endometrial ultrasound & Post-menopause & Every 12 months \\
\hline \multicolumn{3}{|l|}{ Men and women } \\
\hline $\begin{array}{l}\text { Annual physical exam with focus on thyroid } \\
\text { and breast }\end{array}$ & $\begin{array}{l}\text { Age } 18 \text { or } 5 \text { years younger than youngest age of } \\
\text { cancer diagnosis in family. }\end{array}$ & Every 12 months \\
\hline $\begin{array}{l}\text { Urinalysis (consider urine cytology and renal } \\
\text { ultrasound if family history of renal cancer) }\end{array}$ & & Every 12 months \\
\hline $\begin{array}{l}\text { Thyroid ultrasound } \\
\text { Dermatologic exam } \\
\text { Education regarding signs and symptoms of } \\
\text { cancer }\end{array}$ & Age 18 years (baseline) & $\begin{array}{l}\text { Consider every } 12 \text { months } \\
\text { Consider every } 12 \text { months } \\
\text { Every } 12 \text { months }\end{array}$ \\
\hline
\end{tabular}

\section{Proteus syndrome}

Proteus syndrome (PS) was originally described by Cohen and Hayden in 1979, and was given its name by Wiedemann, after the Greek sea god known for his ability to change his shape. ${ }^{44}$ Proteus syndrome is an extremely rare, highly variable disorder of post-natal mosaic growth dysregulation consisting of progressive, asymmetric, and disproportionate overgrowth. The clinical diagnosis of PS remains challenging and controversial, with misdiagnosis a frequent occurrence. ${ }^{45,46}$ PS is characterized by a sporadic, progressive, mosaic distribution of the following: cerebriform connective tissue nevi, epidermal nevi, asymmetric and disproportionate overgrowth involving disparate anatomical structures, dysregulated adipose deposits, ovarian cystadenomas, parotid adenomas, vascular malformations, lung cysts, and facial abnormalities.

The underlying etiology of PS is unclear and its association with PHTS remains controversial. Zhou et al ${ }^{47}$ were the first to report a putative association between PS and PHTS, in that two out of nine patients meeting established diagnostic criteria for PS possessed a germline PTEN mutation. Furthermore, in five individuals with Proteus-like features, who did not meet diagnostic criteria for PS, CS, or BRRS, three were found to have heterozygous germline PTEN mutation. A possible association between PS and heterozygous germline PTEN mutations was also corroborated by other investigators, ${ }^{48}$ who found a germline PTEN mutation in a patient with PS. These findings, however, were called into question by Biesecker et al, ${ }^{49}$ who found no PTEN mutations in 19 subjects with PS, as well as by Thiffault et $a l^{50}$ who found no intra-exonic PTEN mutations by sequence analysis in 6 individuals with 'indisputable' PS.
Given the controversy in associating PTEN mutation with PS, some have speculated that germline PTEN mutations can cause a distinct phenotype with features similar to PS. Happle ${ }^{51,52}$ recently distinguished between the thickened linear Cowden nevus, associated with a loss of the second copy of PTEN, and the pathognomonic linear Proteus nevus, which is typically flat. Caux et $a l^{53}$ described two patients from CS families with germline PTEN mutation who developed segmental overgrowth, vascular and lymphatic malformations, lipomatosis, and linear epidermal nevi reminiscent of PS. In one of these patients, a loss of the second PTEN allele in an atypical segmental lesion was reported. The authors propose the term 'SOLAMEN' syndrome (segmental overgrowth, lipomatosis, arteriovenous malformation and epidermal nevus) to account for these unusual patients. ${ }^{53}$ A conclusive answer regarding PTEN mutations and PS awaits further study of the molecular basis underlying this syndrome.

Other clinical syndromes associated with loss of PTEN In addition to CS, LD, BRRS, and PS, there are other syndromes that may be associated with germline mutation or deletion of the PTEN gene. PTEN mutations have been described in patients with autism spectrum disorders (ASD) and macrocephaly. In an analysis of 88 patients with ASD and macrocephaly, a de novo missense PTEN mutation, D326N, was detected in a 5-year-old boy with autism, severe language delay, macrocephaly, and polydactyly of both feet. The authors conclude that screening for PTEN mutations should be considered in any patient with ASD and macrocephaly. ${ }^{54}$ However, larger studies are needed to validate these findings. A germline PTEN mutation was also reported in an infant with features of VATER (vertebral 
malformations, anal malformations, tracheoesophageal atresia, renal/radial malformations), hydrocephalus, and macrocephaly. ${ }^{55}$

Germline PTEN mutations have been observed in patients with GI hamartoma syndromes not meeting criteria for CS or BRRS. In a study of 49 patients with unexplained GI hamartomas or hyperplastic polyposis, two patients were found to have germline PTEN mutations. One patient previously classified as Peutz-Jeghers syndrome had a PTEN deletion, and an infant with juvenile polyposis syndrome (JPS) had a hemizygous deletion encompassing both the PTEN and bone morphogenic protein receptor 1A (BMPR1A) genes on chromosome 10q23. ${ }^{56}$ Similarly, Van Hattem et al ${ }^{57}$ recently reported on a subset of patients with JPS with large genomic deletions of both BMPR1A and PTEN. With increased understanding of the spectrum of phenotypic features associated with germline PTEN mutations, which can vary widely from classic CS, it is possible that the spectrum of disorders within PHTS will expand. It is unknown whether patients who have germline PTEN mutations but phenotypically deviate from 'classic' CS should be managed according to the established guidelines (Table 3), and should be included in trials investigating novel therapies for PHTS.

\section{Clinical management Screening}

The most critical aspect in the management of patients with CS is heightened cancer surveillance, given that these patients are at increased risk to develop breast, thyroid, endometrial, and possibly other malignancies. Although it is not certain that patients with germline PTEN mutations who do not meet CS diagnostic criteria are at increased risk of developing cancers, Eng et $a l^{1}$ have advocated that all patients with germline PTEN mutation undergo screening according to the NCCN guidelines (Table 3). Despite the logic that underlies this recommendation, it is unlikely that clinical trials will prove the efficacy of screening to reduce cancer mortality in PHTS patients. Nonetheless, screening that is performed as part of a clinical trial could support its wider application and provide opportunities to investigate prognostic features of benign and malignant tissues from these patients.

\section{Surgical management}

Patients with PHTS, particularly those with CS, are at increased risk ofr primary malignancy in certain organs, and similar to other familial cancer syndromes, are at increased risk of secondary malignancies as well. Therefore, prophylactic resection of susceptible organs may decrease the risk of potentially life-threatening malignancies. However, there is no data to support prophylactic surgery to decrease risk of malignancy or mortality, and likely these studies will never transpire because of the rarity of CS.

Screening for breast cancer is particularly challenging in patients with CS because of the simultaneous presence of benign breast lesions such as fibrocystic changes and hamartomas. Annual mammogram and breast MRI beginning at age $30-35$ years as advocated by NCCN (Table 3 ) is likely to detect a high rate of early stage breast cancer. However, these modalities are also likely to yield a high degree of false positive data, which could lead to increased morbidity to CS patients because of unnecessary diagnostic procedures and heightened anxiety. Some women with CS may elect to undergo prophylactic bilateral mastectomy instead. In women who are BRCA1 and BRCA2 mutation carriers, which is associated with a $56-87 \%$ lifetime breast cancer risk, studies have demonstrated a 90\% risk reduction following prophylactic bilateral mastectomy. ${ }^{58,59}$ Women with CS, who carry a 30-50\% lifetime risk of breast cancer, may also derive benefit from prophylactic bilateral mastectomy. The 2007 NCCN guidelines for CS recommend discussion of options for risk reducing mastectomy on a case-by-case basis, and counseling regarding degree of protection, extent of cancer risk, and reconstruction options.

Total thyroidectomy is generally recommended in CS patients who develop thyroid cancer because of the risk of recurrent malignancy and the difficulty of repeat thyroid resections. In CS patients who develop benign thyroid lesions, a complete thyroidectomy is also recommended because of the increased risk of developing subsequent thyroid cancer and the increased morbidity of repeat biopsies to distinguish between benign nodules and malignancy. ${ }^{20}$

As part of endometrial cancer screening, NCCN guidelines recommend annual blind endometrial biopsy for CS women who are pre-menopausal, and endometrial ultrasound for post-menopausal women. These guidelines make no specific recommendation regarding prophylactic hysterectomy. In some patients, such as post-menopausal women, the risk/benefit ratio may favor prophylactic hysterectomy. For patients with LD and dysplastic cerebellar gangliocytomas, surgical resection to alleviate symptoms related to hydrocephalus or mass effect is recommended; however, regrowth of LD lesions following resection has been reported. ${ }^{37}$ Interventional treatment options for benign skin lesions related to PHTS include curettage, cryosurgery, and laser ablation.

\section{Medical management}

Standard medical therapies For women in the general population who are at increased risk of breast cancer, preventive hormonal agents such as tamoxifen or raloxifen can be considered in those who have at least a 1.6\% 5-year risk. ${ }^{60,61}$ These agents could also be considered in women with CS; however, tamoxifen should be administered with 
extreme caution in women who have not undergone hysterectomy, given the potential for additive endometrial cancer risk. There are no proven chemopreventive strategies for thyroid and endometrial carcinoma, nor are there established medical therapies to reduce the growth or size of benign hamartomatous disease.

Experimental therapies A new strategy to control PHTS symptoms, prevent tumor growth, and/or treat established cancers is to test drugs that inhibit the PI3K/Akt/mTOR pathway in PHTS patients, given that loss of PTEN increases activation of the pathway in benign and malignant tissues of patients with PHTS and drives cellular proliferation, migration, and survival. An advantage of this strategy is that the accessibility of skin and GI hamartomas before and after therapy in PHTS patients would provide an opportunity to correlate changes in tumor growth and/or metabolism with changes in phosphorylation of pathway components such as Akt, S6K, S6, and 4E-BP1.

The development of PI3K/Akt/mTOR inhibitors is an area of intense research in oncology, as this pathway promotes cellular survival and chemotherapeutic resistance in a variety of malignancies. Inhibition of proximal components of the pathway such as PI3K or Akt, while potentially desirable, is currently less feasible for patients with PHTS because the results of early phase clinical trials with these inhibitors in cancer patients have not been published. In addition, it is not clear if inhibitors or PI3K or Akt will be as tolerable as inhibitors of mTOR. Inhibitors of mTOR are the most clinically developed, and may serve as viable agents to treat PHTS. Rapamycin, a specific mTOR inhibitor, is FDA approved to prevent transplant rejection and for use in drug-eluting cardiac stents. Temsirolimus, a rapamycin analog, was recently FDA approved for the treatment of advanced renal cell carcinoma.

mTOR inhibitors are promising therapies for PHTS patients because loss of PTEN is a positive predictive factor for response to these drugs. Preclinical data show that tumors with mutant PTEN have increased sensitivity to mTOR inhibitors. Shi et al ${ }^{62}$ demonstrated that PTENdeficient human myeloma cells were over 1000 times more sensitive to temsirolimus than myeloma cells expressing wild-type PTEN. This relative sensitivity to temsirolimus conferred by inactive PTEN has also been observed in mouse xenograft studies, where tumors with inactive PTEN and/or activation of Akt/mTOR pathway respond best to rapamycin analogs. ${ }^{63,64}$ Similarly, Podsypanina et al ${ }^{65}$ demonstrated that PTEN heterozygote mice treated with temsirolimus exhibited diminished tumor burden in many organs through reduction in tumor size and prevention of tumor progression.

The feasibility of using mTOR inhibitors in PHTS is also supported by recent studies in other hamartoma syndromes. Bissler et $a l^{66}$ demonstrated that rapamycin significantly decreased the size of angiomyolipomas and improved pulmonary function in patients with tuberous sclerosis (TSC). Because assessment of mTOR inhibition in affected or surrogate tissues was not reported in this trial, correlations between clinical response and mTOR inhibition cannot be made. With the encouraging clinical results of this study, a multicenter phase III trial is underway to study mTOR inhibition to treat the benign manifestations of TSC. In addition to TSC, the clinical course of a child with Proteus syndrome and a germline PTEN mutation treated with rapamycin has been reported. Rapamycin caused regression of mediastinal and pelvic masses and mesenteric adenopathy, and improved the patient's respiratory distress and nutritional status. ${ }^{67}$ These outcomes lend further support for the rationale to treat patients with germline PTEN mutations with rapamycin, as well as the theory that benign tumors in a subset of PS patients are driven by the loss of PTEN, leading to mTOR activation. Finally, the use of rapamycin is also being studied in neurofibromatosis and Birt-Hogg-Dubé, other disorders in which the loss of different tumor suppressor genes alters signaling pathways that converge to activate mTOR.

\section{Considerations for the use of PI3K/Akt/mTOR inhibitors} in PHTS As it is possible that PHTS patients could benefit from drugs that inhibit the PI3K/Akt/mTOR pathway, this benefit might not be without cost. The risk/benefit ratio may differ for patients with established malignancy versus patients without malignancy, in that toxicities of pathway inhibitors will be more acceptable for patients with lifethreatening, advanced malignancies. Still, the risk could be high for all PHTS patients. The PI3K/Akt/mTOR pathway has a vital function in many normal cellular processes, including endothelial and cardiomyocyte survival, as well as glucose regulation. ${ }^{68,69}$ Because patients with PHTS bear germline PTEN mutations in every cell, pathway inhibitors could have greater toxicity than that observed in cancer patients whose PTEN mutations are limited to only tumor tissues. This concern may be hypothetical as excess toxicity was not observed in the Bissler et al $^{66}$ trial of rapamycin in TSC. An additional argument against using inhibitors of the PI3K/Akt/mTOR pathway in patients with PHTS is that even if these drugs were to compensate for loss of PTEN lipid phosphatase activity, they would not compensate for other functions of PTEN such as protein phosphatase activity and maintenance of chromosomal stability.

Two other considerations apply specifically to mTOR inhibitors. First, the use of mTOR inhibitors can lead to feedback activation of Akt, which could negate any effect of mTOR inhibitors by promoting propagation of the Akt signal to other downstream substrates. ${ }^{70-72}$ This phenomenon was recently demonstrated in a trial of rapamycin in patients with PTEN-deficient glioblastoma, in which feedback activation of Akt was associated with a shorter time to disease progression. ${ }^{73}$ However, it is unclear how widely such feedback will be observed. 
Another concern with rapamycin is that it has a 'black-box' warning regarding the possibility of developing opportunistic infections and lymphomas in the setting of immune suppression. However, the attribution of this risk is based on organ transplant patients receiving rapamycin in combination with cyclosporine and/or corticosteroids. It has yet to be determined whether single-agent therapy with rapamycin or its analogs would produce the same effects on immune function. Reassuringly, no episodes of opportunistic infection were reported in the study of rapamycin in patients with TSC. ${ }^{66}$

\section{Future directions for PHTS}

The future for PHTS patients has great promise because of efforts to improve diagnostic criteria, and the development of new therapeutics that will present challenges to traditional thinking about clinical trial design. Distinctions between the various PHTS syndromes, especially between CS and BRRS, may no longer be clinically relevant. In light of this, an international collaborative effort is underway to prospectively collect genotypic and phenotypic data from thousands of CS patients to identify the simplest means by which to predict germline PTEN mutation. ${ }^{35}$ This analysis may simplify the diagnostic criteria and ultimately group BRRS and CS into one syndrome. Revised diagnostic criteria may also decrease the complexity associated with diagnosing PHTS and ensure that patients with PTEN mutations are better recognized by the medical community so that genotyping can be performed and appropriate cancer surveillance utilized. As the understanding of the spectrum of disorders associated with PTEN germline mutation expands, so too will the understanding of PTEN biology.

Potential therapies for PHTS that are currently available for clinical testing include rapamycin and its analogs that are highly specific for mTOR, but indirectly inhibit mTOR by binding FKBP12. Newer, direct mTOR inhibitors that target the ATP-binding domain are also being developed. ${ }^{74,75}$ These inhibitors will likely have different properties than rapamycin or its analogs. For example, the similarity of structure of the ATP-binding domains in kinases related to mTOR suggests that direct mTOR inhibitors might also inhibit kinases such as class I PI3K isoforms, and DNA repair enzymes such as DNA-PK and ATM. This has the potential advantage of negating feedback Akt activation that might be observed with agents that only inhibit mTOR inhibition. However, such activity may also be associated with greater toxicity than rapamycin, and thus mTOR inhibitors with a wide spectrum of activity should be initially tested only in PHTS patients with advanced malignancy, where the potential benefits may outweigh the risks.

In addition to utilizing inhibitors of the PI3K/Akt/mTOR pathway, future therapies for PHTS may use alternative strategies to restore PTEN function. For example, it might be possible to counteract mutations in PTEN outside the phosphatase domain that lead to increased protein degradation by using a proteosome inhibitor such as bortezomib. Blocking the proteosome would decrease degradation and could increase PTEN protein levels. In patients whose PTEN mutations are outside of exon 5, PTEN phosphatase function might be restored by this approach. In addition, it is possible that gene targets of PTEN outside the canonical PI3K/Akt/mTOR pathway could also be exploited. Such targets could include PINK1, a PTEN-induced protein kinase. Although agonists of PINK1 may prove to be a viable future strategy for treatment of PHTS, none currently exist. In addition, PINK1 has been studied most extensively in Parkinson disease, ${ }^{76}$ and further studies on the function of PINK1 in PHTS and in cancer ${ }^{77}$ are needed.

The development of new therapeutics for PHTS will present challenges for clinical trial design. What is the ideal clinical setting in which to test pathway inhibitors in PHTS patients? A traditional drug-development approach would test molecularly targeted agents exclusively in PHTS patients with advanced incurable malignancies who have progressed after receiving 'standard' chemotherapies or are resistant to standard therapies. There are at least two reasons that testing of these agents should be considered earlier in the disease process, especially for patients who only serve to gain marginal benefit from frontline chemotherapy. First, tumors that develop after PTEN loss may be 'addicted' to the subsequent activation of the PI3K/ Akt/mTOR pathway. Previous examples of 'oncogene addiction' such as bcr-abl in chronic myelogenous leukemia, c-kit in GI stromal tumors, and EGFR in lung cancer strongly suggest that drugs that target these 'addictions' are useful as first-line therapies. It is possible that inhibitors of the PI3K/Akt/mTOR pathway might achieve the same success in tumors from PHTS patients driven by the loss of PTEN. Second, PHTS patients suffer significant morbidity from numerous diagnostic work-ups of tumors that form, and significant anxiety from the knowledge of being at increased risk to develop certain cancers. An effective preventive approach could mitigate both of these burdens. Therefore, drugs that have favorable toxicity profiles should be tested in small, short pilot trials with PHTS patients, even if they do not have cancer. Such trials could provide preliminary evidence of pathway inhibition, tolerability, and inhibition of tumor growth. Positive early trials could then be followed by larger trials to assess longterm effects on toxicity and efficacy. Although preventing cancer is a very difficult benchmark to achieve, PHTS patients are unusual in that their predisposition to cancer is based on a defined molecular change, not environmental exposure or behavior. Adopting different concepts of clinical trial design could identify new drugs for the treatment or prevention of tumors related to germline loss of PTEN, and thereby provide hope to patients with PHTS. 


\section{Conflict of interest}

None declared.

\section{References}

1 Eng C: Will the real Cowden syndrome please stand up: revised diagnostic criteria. J Med Genet 2000; 37: 828-830.

2 Marsh DJ, Coulon V, Lunetta KL et al: Mutation spectrum and genotype-phenotype analyses in Cowden disease and BannayanZonana syndrome, two hamartoma syndromes with germline PTEN mutation. Hum Mol Genet 1998; 7: 507-515.

3 Eng C: PTEN: one gene, many syndromes. Hum Mutat 2003; 22: 183-198.

4 Tamura M, Gu J, Matsumoto K, Aota S, Parsons R, Yamada KM: Inhibition of cell migration, spreading, and focal adhesions by tumor suppressor PTEN. Science 1998; 280: 1614-1617.

5 Simpson L, Parsons R: PTEN: life as a tumor suppressor. Exp Cell Res 2001; 264: 29-41.

6 Weng LP, Smith WM, Brown JL, Eng C: PTEN inhibits insulinstimulated MEK/MAPK activation and cell growth by blocking IRS-1 phosphorylation and IRS-1/Grb-2/Sos complex formation in a breast cancer model. Hum Mol Genet 2001; 10: 605-616.

7 Shen WH, Balajee AS, Wang J et al: Essential role for nuclear PTEN in maintaining chromosomal integrity. Cell 2007; 128: 157-170.

8 Di Cristofano A, Pesce B, Cordon-Cardo C, Pandolfi PP: Pten is essential for embryonic development and tumour suppression. Nat Genet 1998; 19: 348-355.

9 Suzuki A, de la Pompa JL, Stambolic V et al: High cancer susceptibility and embryonic lethality associated with mutation of the PTEN tumor suppressor gene in mice. Curr Biol 1998; 8: $1169-1178$.

10 Podsypanina K, Ellenson LH, Nemes A et al: Mutation of Pten/ Mmac1 in mice causes neoplasia in multiple organ systems. Proc Natl Acad Sci USA 1999; 96: 1563-1568.

11 Furnari FB, Lin H, Huang HS, Cavenee WK: Growth suppression of glioma cells by PTEN requires a functional phosphatase catalytic domain. Proc Natl Acad Sci USA 1997; 94: 12479-12484.

12 Davies MA, Kim SJ, Parikh NU, Dong Z, Bucana CD, Gallick GE: Adenoviral-mediated expression of MMAC/PTEN inhibits proliferation and metastasis of human prostate cancer cells. Clin Cancer Res 2002; 8: 1904-1914.

13 Stambolic V, Suzuki A, de la Pompa JL et al: Negative regulation of PKB/Akt-dependent cell survival by the tumor suppressor PTEN. Cell 1998; 95: 29-39.

14 Lloyd II KM, Dennis M: Cowden's disease. A possible new symptom complex with multiple system involvement. Ann Intern Med 1963; 58: 136-142.

15 Liaw D, Marsh DJ, Li J et al: Germline mutations of the PTEN gene in Cowden disease, an inherited breast and thyroid cancer syndrome. Nat Genet 1997; 16: 64-67.

16 Starink TM, van der Veen JP, Arwert $\mathrm{F}$ et al: The Cowden syndrome: a clinical and genetic study in 21 patients. Clin Genet 1986; 29: 222-233.

17 McGarrity TJ, Wagner Baker MJ, Ruggiero FM et al: GI polyposis and glycogenic acanthosis of the esophagus associated with PTEN mutation positive Cowden syndrome in the absence of cutaneous manifestations. Am J Gastroenterol 2003; 98: 1429-1434.

18 Harach HR, Soubeyran I, Brown A, Bonneau D, Longy M: Thyroid pathologic findings in patients with Cowden disease. Ann Diagn Pathol 1999; 3: 331-340.

19 Schrager CA, Schneider D, Gruener AC, Tsou HC, Peacocke M: Clinical and pathological features of breast disease in Cowden's syndrome: an underrecognized syndrome with an increased risk of breast cancer. Hum Pathol 1998; 29: 47-53.

20 Gustafson S, Zbuk KM, Scacheri C, Eng C: Cowden syndrome. Semin Oncol 2007; 34: 428-434.

21 Tan WH, Baris HN, Burrows PE et al: The spectrum of vascular anomalies in patients with PTEN mutations: implications for diagnosis and management. J Med Genet 2007; 44: 594-602.
22 Lachlan KL, Lucassen AM, Bunyan D, Temple IK: Cowden syndrome and Bannayan Riley Ruvalcaba syndrome represent one condition with variable expression and age-related penetrance: results of a clinical study of PTEN mutation carriers. J Med Genet 2007; 44: 579-585.

23 Longy M, Lacombe D: Cowden disease. Report of a family and review. Ann Genet 1996; 39: 35-42.

24 Nusbaum R, Vogel KJ, Ready K: Susceptibility to breast cancer: hereditary syndromes and low penetrance genes. Breast Dis 2006; 27: $21-50$.

25 Fackenthal JD, Marsh DJ, Richardson AL et al: Male breast cancer in Cowden syndrome patients with germline PTEN mutations. J Med Genet 2001; 38: 159-164.

26 Pilarski R, Eng C: Will the real Cowden syndrome please stand up (again)? Expanding mutational and clinical spectra of the PTEN hamartoma tumour syndrome. J Med Genet 2004; 41: 323-326.

27 Marsh DJ, Kum JB, Lunetta KL et al: PTEN mutation spectrum and genotype-phenotype correlations in Bannayan-Riley-Ruvalcaba syndrome suggest a single entity with Cowden syndrome. Hum Mol Genet 1999; 8: 1461-1472.

28 Zhou XP, Waite KA, Pilarski R et al: Germline PTEN promoter mutations and deletions in Cowden/Bannayan-Riley-Ruvalcaba syndrome result in aberrant PTEN protein and dysregulation of the phosphoinositol-3-kinase/Akt pathway. Am J Hum Genet 2003; 73: 404-411.

29 Yang $\mathrm{H}$, Kong W, He L et al: MicroRNA expression profiling in human ovarian cancer: miR-214 induces cell survival and cisplatin resistance by targeting PTEN. Cancer Res 2008; 68: $425-433$.

30 Trotman LC, Wang X, Alimonti A et al: Ubiquitination regulates PTEN nuclear import and tumor suppression. Cell 2007; 128: $141-156$.

31 Wang X, Trotman LC, Koppie T et al: NEDD4-1 is a protooncogenic ubiquitin ligase for PTEN. Cell 2007; 128: 129-139.

32 Mirmohammadsadegh A, Marini A, Nambiar S et al: Epigenetic silencing of the PTEN gene in melanoma. Cancer Res 2006; 66: $6546-6552$.

33 Sarquis MS, Agrawal S, Shen L, Pilarski R, Zhou XP, Eng C: Distinct expression profiles for PTEN transcript and its splice variants in Cowden syndrome and Bannayan-Riley-Ruvalcaba syndrome. Am J Hum Genet 2006; 79: 23-30.

34 Agrawal S, Eng C: Differential expression of novel naturally occurring splice variants of PTEN and their functional consequences in Cowden syndrome and sporadic breast cancer. Hum Mol Genet 2006; 15: 777-787.

35 Zbuk KM, Eng C: Cancer phenomics: RET and PTEN as illustrative models. Nat Rev Cancer 2007; 7: 35-45.

36 Zhou XP, Marsh DJ, Morrison CD et al: Germline inactivation of PTEN and dysregulation of the phosphoinositol-3-kinase/Akt pathway cause human Lhermitte-Duclos disease in adults. Am J Hum Genet 2003; 73: 1191-1198.

37 Williams III DW, Elster AD, Ginsberg LE, Stanton C: Recurrent Lhermitte-Duclos disease: report of two cases and association with Cowden's disease. AJNR Am J Neuroradiol 1992; 13: 287-290.

38 Abel TW, Baker SJ, Fraser MM et al: Lhermitte-Duclos disease: a report of 31 cases with immunohistochemical analysis of the PTEN/AKT/mTOR pathway. J Neuropathol Exp Neurol 2005; 64: 341-349.

39 Bannayan GA: Lipomatosis, angiomatosis, and macrencephalia. A previously undescribed congenital syndrome. Arch Pathol 1971; 92: $1-5$.

40 Moretti-Ferreira D, Koiffmann CP, Souza DH, Diament AJ, Wajntal A: Macrocephaly, multiple lipomas, and hemangiomata (Bannayan-Zonana syndrome): genetic heterogeneity or autosomal dominant locus with at least two different allelic forms? Am J Med Genet 1989; 34: 548-551.

41 Miles JH, Zonana J, McFarlane J, Aleck KA, Bawle E: Macrocephaly with hamartomas: Bannayan-Zonana syndrome. Am J Med Genet 1984; 19: $225-234$. 
42 Hayashi Y, Ohi R, Tomita Y, Chiba T, Matsumoto Y: BannayanZonana syndrome associated with lipomas, hemangiomas, and lymphangiomas. J Pediatr Surg 1992; 27: 722-723.

43 Parisi MA, Dinulos MB, Leppig KA, Sybert VP, Eng C, Hudgins L: The spectrum and evolution of phenotypic findings in PTEN mutation positive cases of Bannayan-Riley-Ruvalcaba syndrome. I Med Genet 2001; 38: 52-58.

44 Wiedemann HR, Burgio GR, Aldenhoff P, Kunze J, Kaufmann HJ, Schirg E: The proteus syndrome. Partial gigantism of the hands and/or feet, nevi, hemihypertrophy, subcutaneous tumors, macrocephaly or other skull anomalies and possible accelerated growth and visceral affections. Eur J Pediatr 1983; 140: 5-12.

45 Cohen MM, Jr: Proteus syndrome: an update. Am J Med Genet C Semin Med Genet 2005; 137: 38-52.

46 Cohen Jr MM, Turner JT, Biesecker LG: Proteus syndrome: misdiagnosis with PTEN mutations. Am J Med Genet A 2003; 122: $323-324$

47 Zhou $\mathrm{X}$, Hampel $\mathrm{H}$, Thiele $\mathrm{H}$ et al: Association of germline mutation in the PTEN tumour suppressor gene and Proteus and Proteus-like syndromes. Lancet 2001; 358: 210-211.

48 Smith JM, Kirk EP, Theodosopoulos G et al: Germline mutation of the tumour suppressor PTEN in Proteus syndrome. J Med Genet 2002; 39: 937 - 940 .

49 Biesecker LG, Rosenberg MJ, Vacha S, Turner JT, Cohen MM: PTEN mutations and proteus syndrome. Lancet 2001; 358: 2079-2080.

50 Thiffault I, Schwartz CE, Der Kaloustian V, Foulkes WD: Mutation analysis of the tumor suppressor PTEN and the glypican 3 (GPC3) gene in patients diagnosed with Proteus syndrome. Am J Med Genet A 2004; 130: 123-127.

51 Happle R: Type 2 segmental Cowden disease $v s$ Proteus syndrome. Br J Dermatol 2007; 156: 1089-1090.

52 Happle R: Linear Cowden nevus: a new distinct epidermal nevus. Eur J Dermatol 2007; 17: 133-136.

53 Caux F, Plauchu H, Chibon F et al: Segmental overgrowth, lipomatosis, arteriovenous malformation and epidermal nevus (SOLAMEN) syndrome is related to mosaic PTEN nullizygosity. Eur J Hum Genet 2007; 15: 767-773.

54 Buxbaum JD, Cai G, Chaste P et al: Mutation screening of the PTEN gene in patients with autism spectrum disorders and macrocephaly. Am J Med Genet B Neuropsychiatr Genet 2007; 144: $484-491$

55 Reardon W, Zhou XP, Eng C: A novel germline mutation of the PTEN gene in a patient with macrocephaly, ventricular dilatation, and features of VATER association. J Med Genet 2001; 38: $820-823$

56 Sweet K, Willis J, Zhou XP et al: Molecular classification of patients with unexplained hamartomatous and hyperplastic polyposis. JAMA 2005; 294: 2465-2473.

57 van Hattem WA, Brosens LA, de Leng WW et al: Large genomic deletions of Smad4, Bmpr1a and Pten in juvenile polyposis. Gut 2008; 57 (5): 623-627.

58 Meijers-Heijboer H, van Geel B, van Putten WL et al: Breast cancer after prophylactic bilateral mastectomy in women with a BRCA1 or BRCA2 mutation. N Engl J Med 2001; 345: 159-164.

59 Hartmann LC, Sellers TA, Schaid DJ et al: Efficacy of bilateral prophylactic mastectomy in BRCA1 and BRCA2 gene mutation carriers. J Natl Cancer Inst 2001; 93: 1633-1637.

60 Fisher B, Costantino JP, Wickerham DL et al: Tamoxifen for the prevention of breast cancer: current status of the National
Surgical Adjuvant Breast and Bowel Project P-1 study. I Natl Cancer Inst 2005; 97: 1652-1662.

61 Vogel VG, Costantino JP, Wickerham DL et al: Effects of tamoxifen vs raloxifene on the risk of developing invasive breast cancer and other disease outcomes: the NSABP Study of Tamoxifen and Raloxifene (STAR) P-2 trial. JAMA 2006; 295: $2727-2741$

62 Shi Y, Gera J, Hu L et al: Enhanced sensitivity of multiple myeloma cells containing PTEN mutations to CCI-779. Cancer Res 2002; 62: $5027-5034$.

63 Neshat MS, Mellinghoff IK, Tran C et al: Enhanced sensitivity of PTEN-deficient tumors to inhibition of FRAP/mTOR. Proc Natl Acad Sci USA 2001; 98: 10314-10319.

$64 \mathrm{Yu} \mathrm{K}$, Toral-Barza L, Discafani C et al: mTOR, a novel target in breast cancer: the effect of CCI-779, an mTOR inhibitor, in preclinical models of breast cancer. Endocr Relat Cancer 2001; 8: 249-258.

65 Podsypanina K, Lee RT, Politis C et al: An inhibitor of mTOR reduces neoplasia and normalizes p70/S6 kinase activity in Pten $^{+/-}$mice. Proc Natl Acad Sci USA 2001; 98: 10320-10325.

66 Bissler JJ, McCormack FX, Young LR et al: Sirolimus for angiomyolipoma in tuberous sclerosis complex or lymphangioleiomyomatosis. $N$ Engl J Med 2008; 358: 140-151.

67 Marsh DJ, Trahair TN, Martin JL et al: Rapamycin treatment for a child with germline PTEN mutation. Nat Clin Pract Oncol 2008; 5: $357-361$.

68 Fujio Y, Nguyen T, Wencker D, Kitsis RN, Walsh K: Akt promotes survival of cardiomyocytes in vitro and protects against ischemiareperfusion injury in mouse heart. Circulation 2000; 101: 660-667.

69 Zdychova J, Komers R: Emerging role of Akt kinase/protein kinase B signaling in pathophysiology of diabetes and its complications. Physiol Res 2005; 54: 1-16.

70 Sun SY, Rosenberg LM, Wang X et al: Activation of Akt and eIF4E survival pathways by rapamycin-mediated mammalian target of rapamycin inhibition. Cancer Res 2005; 65: 7052-7058.

71 O'Reilly KE, Rojo F, She QB et al: mTOR inhibition induces upstream receptor tyrosine kinase signaling and activates Akt. Cancer Res 2006; 66: 1500-1508.

72 Wan X, Harkavy B, Shen N, Grohar P, Helman LJ: Rapamycin induces feedback activation of Akt signaling through an IGF-1Rdependent mechanism. Oncogene 2007; 26: 1932-1940.

73 Cloughesy TF, Yoshimoto K, Nghiemphu P et al: Antitumor activity of rapamycin in a phase I trial for patients with recurrent PTEN-deficient glioblastoma. PLoS Med 2008; 5: e8.

74 Fan QW, Cheng CK, Nicolaides TP et al: A dual phosphoinositide3-kinase alpha/mTOR inhibitor cooperates with blockade of epidermal growth factor receptor in PTEN-mutant glioma. Cancer Res 2007; 67: 7960-7965.

75 Papadopoulos KP, Markman B, Tabernero J et al: A phase I doseescalation study of the safety, parmacokinetics (PK), and pharmacodynamics (PD) of a novel PI3K inhibitor, XL765, administered orally to patients (pts) with advanced solid tumors. J Clin Oncol 2008; 26: (20 May suppl; abstract 3510).

76 Beilina A, Van Der Brug M, Ahmad R et al: Mutations in PTENinduced putative kinase 1 associated with recessive parkinsonism have differential effects on protein stability. Proc Natl Acad Sci USA 2005; 102: 5703-5708.

77 Unoki M, Nakamura Y: Growth-suppressive effects of BPOZ and EGR2, two genes involved in the PTEN signaling pathway. Oncogene 2001; 20: 4457-4465. 